\title{
First steps towards a sentinel network for Huanglongbing disease outbreak detection in the Mediterranean basin
}

Among the world's citrus countries, so far only Australia and the Mediterranean basin are still Huanglongbing (HLB)-free. With a yearly production of $18 \mathrm{Mt}$ of citrus, the Mediterranean basin belongs to the three major citrusproducing areas. But HLB may appear sooner or later: trade, tourism and pilgrimages constitute a significant risk to Citrus production. The threat could come:

- from the Atlantic Islands: T. erytreae was reported in Madeira and the Canary Islands - about $500 \mathrm{~km}$ from Agadir in Morocco - ten years ago,

- from Asia: the threat could come from the East (Saudi Arabia, Iran, Yemen),

- from the South: Ethiopia, Sudan, Somalia.

A training course, jointly organized by Cirad (France), Embrapa (Brazil) and the University of Yaoundé I (Cameroon) and jointly funded by the Agropolis Foundation (France) and Capes (Brazil), was held at CIRAD Montpellier, France (5th-8th April, 2011), on HLB symptoms, psyllid identification (Diaphorina citri and Trioza erytreae) and molecular diagnosis. This event gathered Moroccan, Tunisian, Egyptian and Turkish partners involved in the citrus sector or in plant pathology research.

This event led to the foundation of a sentinel network in the South Mediterranean basin aimed at defining and implementing actions for a prevention strategy and education. The network must be extended to the entire Mediterranean basin (southern Europe, Near East), everywhere there are Citrus trees. The goal is that national plant protection services must be ready to eradicate any outbreak of HLB or psyllid vector invasion at its earliest stage. The later the detection is made, the more disastrous the consequences. The group decided several actions to be undertaken as soon as possible, such as, first of all, the quarantine services of all the Mediterranean countries registering Trioza erytraea, Diaphorina citri and Candidatus Liberibacter spp. as quarantine organisms.

A steering committee has been elected:

Baysal Ömür, Turkey, baysalo@yahoo.de; Cherif Mohamed, Tunisia, cherifmoh2003@yahoo.fr; Dollet Michel, France, michel.dollet@cirad.fr; Ferraz Laranjeira Franscisco, Brazil, chico@cnpmf.embrapa.br); Sahar A. Youssef, Egypt, saharyoussef@link.net); Tamesse Joseph Lebel, Cameroon, jltamesse@yahoo.fr); Zemzami Mustapha, Morocco,zemzami@domaines.co.ma)

(Sources: CIRAD, EMBRAPA)

Additional information will be available in FruiTrop n ${ }^{\circ} 189$ (May 2011). 


\section{Actualités}

\section{Premiers pas vers un réseau d'épidémiosurveillance du greening en Méditerranée}

La Méditerranée est la seule zone au monde avec l'Australie à être épargnée par le greening. Cependant, les échanges commerciaux, les flux de touristes ou les pèlerinages constituent des risques importants d'introduction. La menace vient des îles de l'Atlantique, où Trioza erytreae a été identifié depuis dix ans (Madère et Canaries). Elle vient aussi de pays où la maladie et/ou les vecteurs sont présents, situés à l'Est (Iran, Yémen et Arabie saoudite), ou au Sud (Ethiopie, Soudan et Somalie).

Un atelier de formation organisé conjointement par le CIRAD (France), l'EMBRAPA (Brésil) et l'université de Yaoundé (Cameroun) et financé par la Fondation Agropolis (France) et le Capes (Brésil) s'est tenu à Montpellier du 5 au 8 avril sur les symptômes du greening, l'identification des vecteurs et le diagnostic moléculaire. Il a rassemblé des chercheurs en pathologie végétale et des professionnels de la filière citricole du Maroc, de Tunisie, d'Egypte et de Turquie.

Un embryon de réseau d'épidémiosurveillance du sud du Bassin méditerranéen a été constitué, afin de définir et mettre en place une stratégie de formation et de prévention. Ce réseau doit maintenant être étendu au reste du Bassin méditerranéen pour que les services nationaux de protection des végétaux puissent être prêts à éradiquer au plus vite - afin d'en limiter les conséquences - toute introduction de la maladie ou de son vecteur. La reconnaissance de T. erytreae, Diaphorina citri et Canditatus liberibacter comme organismes de quarantaine par les services sanitaires de tous les pays méditerranéens a été définie comme la première action prioritaire.

Pour plus d'informations, lire FruiTrop n ${ }^{\circ} 189$ (mai 2011).

(Sources : CIRAD, EMBRAPA) 